\title{
BMJ Open Effectiveness of workplace exercise interventions in the treatment of musculoskeletal disorders in office workers: a protocol of a systematic review
}

\author{
Carlos Tersa-Miralles (D) , ${ }^{1}$ Roland Pastells-Peiró (D) , ${ }^{1,2}$ \\ Francesc Rubí-Carnacea (D) , 1,2,3 Filip Bellon (D) , 1,2 Esther Rubinat Arnaldo
}

To cite: Tersa-Miralles C, Pastells-Peiró R, RubíCarnacea F, et al. Effectiveness of workplace exercise interventions in the treatment of musculoskeletal disorders in office workers: a protocol of a systematic review. BMJ Open 2020;10:e038854. doi:10.1136/ bmjopen-2020-038854

- Prepublication history and additional material for this paper are available online. To view these files, please visit the journal online (http://dx.doi. org/10.1136/bmjopen-2020038854).

Received 17 April 2020

Revised 11 November 2020

Accepted 23 November 2020

Check for updates

(c) Author(s) (or their employer(s)) 2020. Re-use permitted under CC BY-NC. No commercial re-use. See rights and permissions. Published by BMJ.

${ }^{1}$ Department of Nursing and Physiotherapy, University of Lleida, Lleida, Spain

${ }^{2}$ Grup de Recerca de Cures en Salut, IRBLleida, Lleida Institute for Biomedical Research Dr. Pifarré Foundation, Lleida, Spain ${ }^{3}$ Grupo de Estudios Sociedad, Salud, Educación y Cultura, University of Lleida, Lleida,

Spain

${ }^{4}$ Center for Biomedical Research on Diabetes and Associated Metabolic Diseases, Instituto de Salud Carlos III, Barcelona, Spain

Correspondence to

Dra Esther Rubinat Arnaldo;

esther.rubinat@udl.cat

\section{ABSTRACT}

Introduction Physical inactivity due to changes in our society towards more sedentary behaviours is leading to health problems. Increasing physical activity might be a good strategy to improve physical strength and reduce the prevalence of illnesses associated with prolonged sitting. Office workers exhibit a sedentary lifestyle with short rest periods or even without pauses during the workday. It is important to perform workplace interventions to treat musculoskeletal disorders caused by prolonged sitting and lack of movement adopted on the office setting. This article describes a protocol for a systematic review to evaluate the effectiveness of exercise interventions on office workers in their work environment.

Methods and analysis A literature search will be performed in the PubMed, CINAHL Plus, Cochrane Library, Scopus, ISI WoS and PeDRO databases for randomised controlled trials and studies published from 1 January 2010 to 31 July 2020 in English or Spanish. The participants will be office workers who spend most of their work time in a sitting position. The interventions performed will include any type of exercise intervention in the workplace. The outcome measures will vary in accordance with the aim of the intervention observed. The results of the review and the outcomes from the studies reviewed will be summarised with a narrative synthesis. The review protocol was developed according to the Preferred Reporting Items for Systematic Review and Meta-Analysis Protocols guidelines.

Ethics and dissemination Ethical approval is not required. The review outcomes and the additional data obtained will be disseminated through publications and in scientific conferences.

PROSPERO registration number CRD42020177462.

\section{INTRODUCTION}

As a result of technological advances being introduced in people's daily lives, our society has changed greatly by adopting an increasingly sedentary lifestyle, leading to prolonged sitting for an entire day. ${ }^{12}$ In addition, this trend is even more impactful when considering the prolonged lifespan of people. The sedentary behaviour in conjunction with the physical inactivity of the population is closely related
Strengths and limitations of this study

- This systematic review protocol follows the Preferred Reporting Items for Systematic Review and MetaAnalysis Protocols guidelines.

- The search strategy was developed in collaboration with an expert documentalist and will include the following databases: PubMed, CINAHL Plus, Cochrane, Scopus, ISI WoS and PeDRO.

- The researcher will conduct a blinded peer review to ensure rigorous and consistent sets of inclusion and exclusion criteria.

- This is an innovative review, as it focuses only on exercise interventions in employees' own workplaces, providing specific data on the most effective active pauses (volume, intensity, time).

- The review may be limited by the heterogeneity of study methodologies and outcome measures due to the diverse types of exercise interventions introduced to office workers.

to the increased prevalence of illnesses due to being both risk factors of health. ${ }^{34}$ The average daily time spent by the adult population in a seated position is $346 \mathrm{~min}$ per day. ${ }^{5}$ More than $30 \%$ of adults worldwide suffer from physical inactivity with higher proportions approximately $43 \%$ in American and eastern Mediterranean countries. ${ }^{6}$

Half of the population, who are of working age, are employed in jobs that do not require any physical activity, such as those in administration or business fields; thus, it is important to spend some time exercising daily to prevent all the pathologies caused by poor postures, prolonged sitting and a lack of movement in the workday. ${ }^{7}$ In an office setting, workers who mainly interact with the computer are predisposed to musculoskeletal disorders due to repetitive movements, static postures and lack of physical activity. ${ }^{8}$ Also, employees working overtime and making work-related decisions have a higher prevalence of suffering from 
musculoskeletal complaints in the neck, shoulder and back region than office workers with fixed schedules and jobs established by their bosses. ${ }^{9}$ Further, workers who perform demanding tasks with little flexibility in their schedules and with short pauses or even a lack of breaks, more commonly develop musculoskeletal low back pain than those who have longer breaks in their workday, such as a lunch break. ${ }^{10}$ Prolonged sitting periods also increase muscle stiffness of the trunk and neck of office workers. As such, an office workplace is an unfavourable environment in terms of the high sedentary behaviour. ${ }^{11}$

Having a daily schedule with different types of activities, such as exercise interventions in the workplace, might help reduce the time workers spend sitting and increase the amount of low-intensity physical activity that employees perform on a daily basis. ${ }^{12}$ For example, breaks with exercises are good options for workplace interventions, which can not only prevent cardiovascular and metabolic illnesses, but also play an important role in the treatment of musculoskeletal dysfunction of the back. ${ }^{13}$

There are a wide variety of types of workplace exercise interventions, such as short breaks throughout the day, and even performing exercise sessions at the start or the end of the workday. The common interventions in the office setting are stretching or strength exercise routines involving the whole body ${ }^{14}$ or focusing on a specific region such as neck/shoulder ${ }^{15}$ trunk or the lower limbs. ${ }^{16}$

Waongenngarm $e t a l^{17}$,s systematic review found positive effects of active breaks with postural change for pain and discomfort of low back pain, without affecting the productivity of office workers. This study conducted on physical exercise in office workers states that the trials reviewed include a wide variety of interventions regarding the duration of the active break, the type of exercise performed or the length of the physical programme at the workplace.

According to Sihawong et al ${ }^{18}$, s systematic review, strength and aerobic exercises can reduce the intensity, disability and duration of neck and shoulder pain caused by poor postures and can be easily performed in work environments because they do not require equipment and can be performed with individuals' own body weight. Other studies $^{19-21}$ examining workplace exercise interventions among symptomatic office workers with musculoskeletal disorders demonstrated a decrease in pain perception.

The aforementioned systematic reviews, which focused on the treatment of musculoskeletal pain in office workers, have been published in recent years evaluating different types of exercise interventions, ${ }^{15}$ or evaluating various kinds of breaks at work. ${ }^{14}$ However, no reviews have focused exclusively on workplace exercise interventions, which are becoming a trend that multinational corporations are implementing with their workers.

The practical implications of this review are aiming to identify standards/patterns in the workplace in order to carry out tailored interventions that are as effective as possible, for the subsequent conduction of a randomised controlled trial and to improve the evidence on physical exercise interventions at work in office workers.

\section{Objectives}

The aim of this study is to present a protocol paper for a systematic review to evaluate the effectiveness of exercise interventions on office workers in their work environment on the treatment of musculoskeletal disorders.

\section{METHODS}

This review protocol follows the recommendations of the Preferred Reporting Items for Systematic Reviews and MetaAnalyses for Protocols (PRISMA-P) reporting guidelines ${ }^{22}$ and the findings will be reported in accordance with the PRISMA guidelines. ${ }^{23}$ This systematic review is registered on the Prospective Register of Systematic reviews.

\section{Criteria for study inclusion \\ Study characteristics}

Randomised controlled studies that have at least one group involved in any kind of intervention with physical exercise in the workplace during the working day.

Randomised controlled studies that have at least one group involved in an intervention with physical exercise in the workplace during the workday and performing a low to moderate physical exercise programme will be included. All studies with ergonomic interventions will be excluded except of posture corrections or patterns taking place during the workday. The search for publications will be restricted considering the temporary interval from 1 January 2010 until 31 July 2020. Studies published in English and Spanish will be considered for inclusion, excluding publications written in other languages. Other sources of research information will be excluded such as books, theses, conferences proceedings, reports or scientific posters.

\section{Participants}

Participants should be office workers who spend most of their work time in a sitting position; have musculoskeletal disorders in all regions of the body or some specific region, such as low back or neck/shoulder; and be capable of performing a low to moderate intensity physical exercise intervention without the need to change clothes or use a sports facility. Studies including participants with other pathologies as neurological deficits, cancer, fractures or an inflammatory process will be excluded.

\section{Interventions}

The included interventions may be any type of exercise programme in the office environment including, essentially, a change from a seated position in front of the computer position to another with a higher physical activity level and performance of any kind of physical exercise, such as stretching, mobility or strength training with or without workout equipment.

\section{Outcomes}

Outcomes will be musculoskeletal disorders measured by different types of validated scales and questionnaires for assessing pain, disfunction or other related aspects for the office workers. Examples of validation measures include 
Table 1 Search terms and PubMed search strategy

\begin{tabular}{|c|c|}
\hline Search terms & PubMed search strategy \\
\hline & 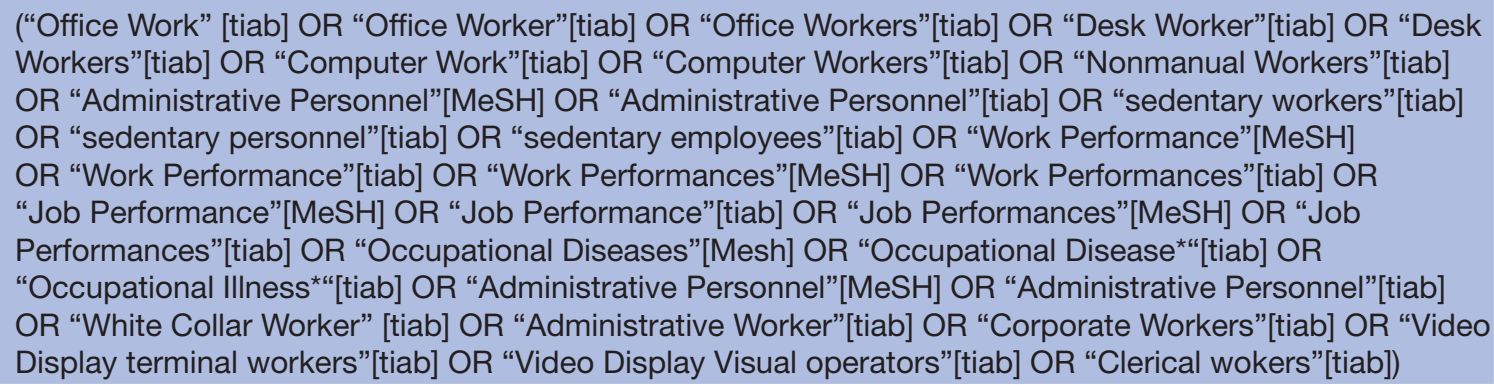 \\
\hline $\begin{array}{l}\text { Musculosk } \\
\text { disorders }\end{array}$ & 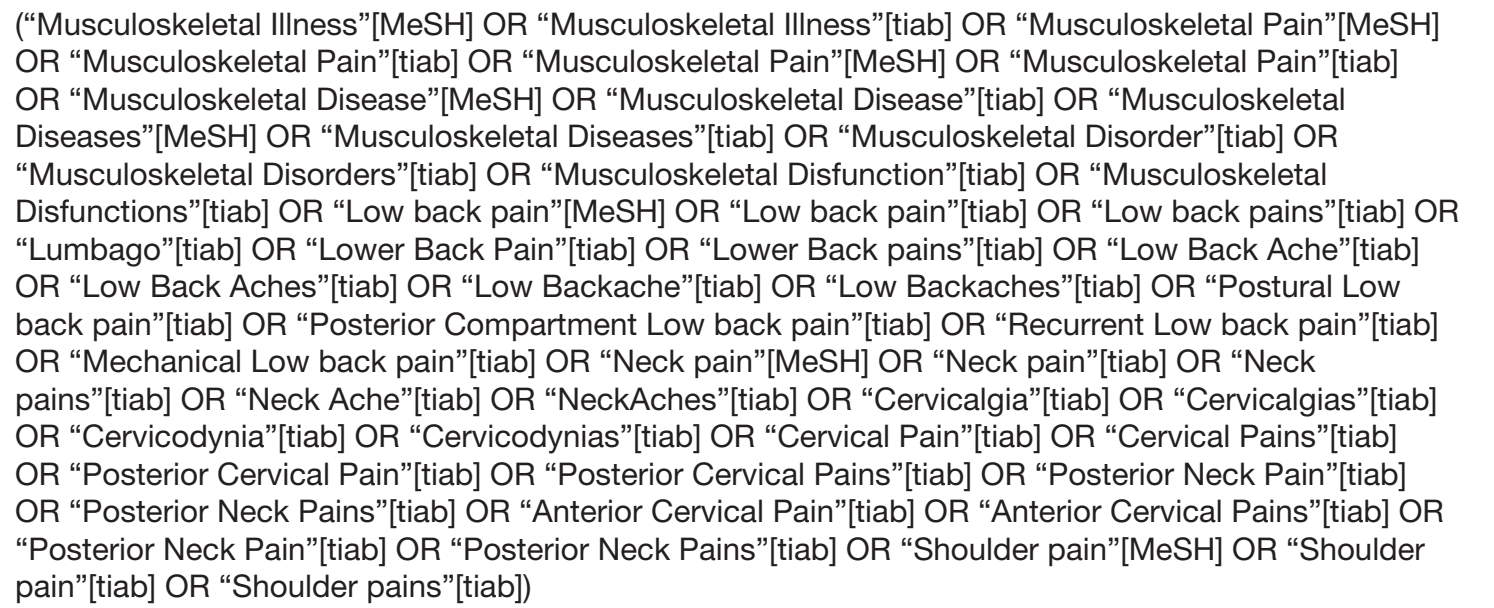 \\
\hline $\begin{array}{l}\text { Exercise } \\
\text { interventions }\end{array}$ & 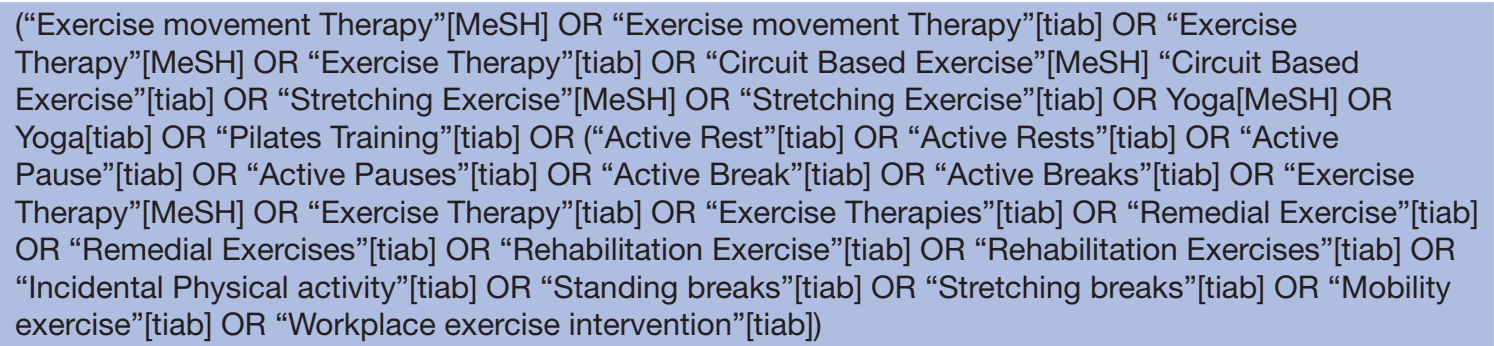 \\
\hline
\end{tabular}

$\mathrm{MeSH}$, medical subject headings.

Scale $^{-1}$ or more specinic scales, such as the Neck Uisability Index for neck pain ${ }^{25}$ or the Oswestry or Roland-Morris Scale $^{26}$ for the measurement of dysfunction caused by low back pain.

\section{Information sources}

For the literature search, the PubMed, CINAHL Plus, Cochrane, Scopus, ISI WoS and PeDRO databases will be searched by using Medical Subject Headings terms and keywords related to office workers, musculoskeletal pain, exercise interventions and adherence. The search strategy in the PubMed database through the MEDLINE nomenclature and thesauruses is available in table 1 .

The search will be adapted and performed in the other databases mentioned above with the aforementioned terminology and search strategy.

\section{Data management and collection}

Once the search has been performed, the results will be imported to EndNote V.X9 $9^{27}$ for duplicates to be removed, and the remaining articles will be subsequently transferred to Covidence ${ }^{28}$ software for screening and data extraction.
'Iwo independent reviewers will screen the titles and abstracts of the articles according to the eligibility criteria to determine the eligibility of the articles. Subsequently, the full texts of the included studies will be reviewed by the same reviewers. If a disagreement between reviewers in any stage of the screening occurs regarding the inclusion of studies, it will be resolved by discussion, and if needed, a third reviewer will determine whether the article will be included.

\section{Data management and risk of bias assessment}

Data from the included studies will be extracted following the recommendations of the Cochrane Handbook for Systematic Reviews of Interventions, ${ }^{29}$ using an adapted data extraction form: the form will follow the steps from the template of the Cochrane good practice data extraction, ${ }^{30}$ taking into account the relevant aspects of the workplace exercise interventions. The data extraction form is shown in online supplemental file 1.

The quality of the studies included in the review will be assessed independently by two reviewers using the Rob2 
tool provided in the Cochrane Reviews to assess the risk of bias, ${ }^{31}$ and a third reviewer will resolve any disagreements in the risk of bias assessment between the two principal reviewers for a final decision.

This tool assesses different aspects of the trials, mainly regarding the design of the methodology and outcome measures. Based on the reviewer's responses to the questions presented for the different domains and a scoring algorithm, each study will be determined to have 'low risk', 'some concerns' or 'high risk' of bias.

\section{Data synthesis}

A narrative synthesis will be conducted in stages, as recommended by the Economic and Social Research Council guidance on the conduct of Narrative Synthesis, ${ }^{32}$ according to the aims of each intervention due to discrepancies in the workplace exercise interventions and the different outcomes included in the studies.

A preliminary synthesis will be performed, presented in a common rubric through tabulation to develop an initial description of the results of included studies. The trials will be grouped by the region of the body that was evaluated. The tables will summarise the results of the interventions with the comparator groups and the outcome measures used in the trials such as scales or questionnaires, also the adherence of the interventions will be described with a numerical percentage of the participants that were lost to follow-up.

Next the relationship between characteristics of individual studies and their reported findings will be explored with a subgroup analysis of the studies included according to each body region categorised from the preliminary synthesis. The assessment of the robustness of the synthesis will be carried with the Rob2 tool.

If the studies included in the review have sufficiently similar characteristics, based on the information in the data extraction form (eg, the use of the same outcome measures such as scales or questionnaires to evaluate pain or dysfunction), a meta-analysis will be performed.

\section{Patient and public involvement}

Data will be extracted from previously published studies. No patients, public institutions or private enterprises of any kind will be involved in this protocol. Additionally, no personal data have been used in the development of this protocol.

\section{Ethics and dissemination}

For the protocol and the posterior systematic review, ethical approval is not required. The aim of this review is to summarise the results of the outcomes from the studies that meet the inclusion criteria.

The review outcomes and the additional data will be disseminated through publications and scientific conferences.

\section{DISCUSSION}

In the 21st century, one of the main reasons that health conditions in society have worsened is due to the increase of physical inactivity. Also, a sedentary lifestyle in the workplace is strongly related to the constant use of electronic devices and technological machinery replacing physical human work. One of the largest groups of people that are affected by this change in society is office workers, as they spend a large number of hours in a seated position. It is necessary to evaluate the evidence found in the trials involving workplace exercise interventions to reduce and treat musculoskeletal pain due to the high prevalence that office workers suffer in their workday.

It is important to analyse the adherence to the exercise interventions as the aim of these exercise programmes is to be performed in the office setting, and the musculoskeletal dysfunction or other work-related stressors might decrease the compliance to realise the physical exercises at the workplace. This can be a potential bias due to the lack of information in the data collection at the end of the intervention due to the loss to follow-up. ${ }^{33-35}$

To the best of our knowledge, this systematic review will be the first published review on the effects of physical exercise in workers' own workplace and during their own working hours, excluding interventions for which the main aim is to perform an ergonomic modification or to set up a 'sit-stand' desk. Both of these types of interventions might increase the physical activity of the workers, but the latter interventions do not involve an exercise programme.

The results of this review will help identify the most effective workplace exercise interventions considering training variables such as the volume, intensity, type of programme and time spent on the exercise per session, allowing to design randomised controlled trial with the best current evidence of workplace intervention in the reduction and treatment of musculoskeletal pain.

Also, this review aims to evaluate the actual evidence on exercise interventions for office workers related to musculoskeletal disorders in their work environment. It is important to improve the workplace health promotion and well-being of the office workers due to the lack of physical activity and the sedentary behaviour due to the requirements of their particular type of job.

Acknowledgements The authors want to acknowledge the Research Group of Health Care (GRECS) —IRB Lleida and the University of Lleida.

Contributors The systematic review protocol was developed and conceived by CTM. RP-P was involved in the review of the manuscript contributing in the methodological aspects and the search strategy. ERA, FRC and FB have been involved in the supervision and quality assurance of the protocol.

Funding The authors have not declared a specific grant for this research from any funding agency in the public, commercial or not-for-profit sectors.

Competing interests None declared.

Patient consent for publication Not required.

Provenance and peer review Not commissioned; externally peer reviewed.

Supplemental material This content has been supplied by the author(s). It has not been vetted by BMJ Publishing Group Limited (BMJ) and may not have been peer-reviewed. Any opinions or recommendations discussed are solely those 
of the author(s) and are not endorsed by BMJ. BMJ disclaims all liability and responsibility arising from any reliance placed on the content. Where the content includes any translated material, BMJ does not warrant the accuracy and reliability of the translations (including but not limited to local regulations, clinical guidelines, terminology, drug names and drug dosages), and is not responsible for any error and/or omissions arising from translation and adaptation or otherwise.

Open access This is an open access article distributed in accordance with the Creative Commons Attribution Non Commercial (CC BY-NC 4.0) license, which permits others to distribute, remix, adapt, build upon this work non-commercially, and license their derivative works on different terms, provided the original work is properly cited, appropriate credit is given, any changes made indicated, and the use is non-commercial. See: http://creativecommons.org/licenses/by-nc/4.0/.

\section{ORCID iDs}

Carlos Tersa-Miralles http://orcid.org/0000-0001-7037-5033

Roland Pastells-Peiró http://orcid.org/0000-0002-9561-9038

Francesc Rubí-Carnacea http://orcid.org/0000-0002-0162-3950

Filip Bellon http://orcid.org/0000-0003-4880-9207

Esther Rubinat Arnaldo http://orcid.org/0000-0003-0232-9777

\section{REFERENCES}

$1 \mathrm{Ng}$ SW, Popkin BM. Time use and physical activity: a shift away from movement across the globe. Obes Rev 2012;13:659-80.

2 Proper KI, Singh AS, van Mechelen W, et al. Sedentary behaviors and health outcomes among adults: a systematic review of prospective studies. Am J Prev Med 2011;40:174-82.

3 de Rezende LFM, Rodrigues Lopes M, Rey-López JP, et al. Sedentary behavior and health outcomes: an overview of systematic reviews. PLoS One 2014;9:e105620.

4 Guthold R, Stevens GA, Riley LM, et al. Worldwide trends in insufficient physical activity from 2001 to 2016: a pooled analysis of 358 population-based surveys with 1.9 million participants. Lancet Glob Health 2018;6:e1077-86.

5 Bauman A, Ainsworth BE, Sallis JF, et al. The descriptive epidemiology of sitting. A 20-country comparison using the International physical activity questionnaire (IPAQ). Am J Prev Med 2011;41:228-35.

6 Hallal PC, Andersen LB, Bull FC, et al. Global physical activity levels: surveillance progress, pitfalls, and prospects. Lancet 2012;380:247-57.

7 Mainenti MRM, Felicio LR, Rodrigues EdeC, et al. Pain, work-related characteristics, and psychosocial factors among computer workers at a university center. J Phys Ther Sci 2014;26:567-73.

8 Collins RM, Janse Van Rensburg DC, Patricios JS. Common workrelated musculoskeletal strains and injuries. South African Family Practice 2011;53:240-6.

9 Mahmud N, Bahari SF, Zainudin NF. Psychosocial and ergonomics risk factors related to neck, shoulder and back complaints among Malaysia office workers. Int J Soc Sci Humanit 2014;4:260-3.

10 Kaliniene G, Ustinaviciene R, Skemiene L, et al. Associations between musculoskeletal pain and work-related factors among public service sector computer workers in Kaunas County, Lithuania. BMC Musculoskelet Disord 2016;17:420.

11 Ryan CG, Dall PM, Granat MH, et al. Sitting patterns at work: objective measurement of adherence to current recommendations. Ergonomics 2011;54:531-8.

12 Parry S, Straker L, Gilson ND, et al. Participatory workplace interventions can reduce sedentary time for office workers--a randomised controlled trial. PLoS One 2013;8:e78957.

13 Kett AR, Sichting F. Sedentary behaviour at work increases muscle stiffness of the back: why roller massage has potential as an active break intervention. Appl Ergon 2020;82:102947.

14 Andersen LL, Christensen KB, Holtermann A, et al. Effect of physical exercise interventions on musculoskeletal pain in all body regions among office workers: a one-year randomized controlled trial. Man Ther 2010;15:100-4.

15 Lidegaard M, Jensen RB, Andersen $\mathrm{CH}$, et al. Effect of brief daily resistance training on occupational neck/shoulder muscle activity in office workers with chronic pain: randomized controlled trial. Biomed Res Int 2013;2013:1-11.

16 Phattharasupharerk S, Purepong N, Eksakulkla S, et al. Effects of Qigong practice in office workers with chronic non-specific low back pain: a randomized control trial. J Bodyw Mov Ther 2019;23:375-81.

17 Waongenngarm P, Areerak K, Janwantanakul P. The effects of breaks on low back pain, discomfort, and work productivity in office workers: a systematic review of randomized and non-randomized controlled trials. Appl Ergon 2018;68:230-9.

18 Sihawong R, Janwantanakul P, Sitthipornvorakul E, et al. Exercise therapy for office workers with nonspecific neck pain: a systematic review. J Manipulative Physiol Ther 2011;34:62-71.

19 de Freitas-Swerts FCT, Robazzi MLdoCC. The effects of compensatory workplace exercises to reduce work-related stress and musculoskeletal pain. Rev Lat Am Enfermagem 2014;22:629-36.

20 Macedo AC, Trindade CS, Brito AP, et al. On the effects of a workplace fitness program upon pain perception: a case study encompassing office workers in a Portuguese context. J Occup Rehabil 2011;21:228-33.

21 Marangoni AH. Effects of intermittent stretching exercises at work on musculoskeletal pain associated with the use of a personal computer and the influence of media on outcomes. Work 2010;36:27-37.

22 Shamseer L, Moher D, Clarke M, et al. Preferred reporting items for systematic review and meta-analysis protocols (PRISMA-P) 2015: elaboration and explanation. BMJ 2015;349:g7647.

23 Moher D, Shamseer L, Clarke M, et al. Preferred reporting items for systematic review and meta-analysis protocols (PRISMA-P) 2015 statement. Syst Rev 2015;4:1

24 Hawker GA, Mian S, Kendzerska T, et al. Measures of adult pain: visual analog scale for pain (vas pain), numeric rating scale for pain (NRS pain), McGill pain questionnaire (MPQ), short-form McGill pain questionnaire (SF-MPQ), chronic pain grade scale (CpGs), short Form36 bodily pain scale (SF-36 BPs), and measure of intermittent and constant osteoarthritis pain (ICOAP). Arthritis Care Res 2011;63 Suppl 11:S240-52.

25 Shin YJ, Kim WH, Kim SG. Correlations among visual analogue scale, neck disability index, shoulder joint range of motion, and muscle strength in young women with forward head posture. $J$ Exerc Rehabil 2017;13:413-7.

26 Chiarotto A, Maxwell LJ, Terwee CB, et al. Roland-Morris disability questionnaire and Oswestry disability index: which has better measurement properties for measuring physical functioning in nonspecific low back pain? systematic review and meta-analysis. Phys Ther 2016;96:1620-37.

27 EndNote [program]. X9.2 version. New York: Clarivate Analytics, 2020.

28 Covidence. Covidence, 2019. Available: https://www.covidence.org/ home

29 Higgins JPT, Green S, Cochrane C. Cochrane Handbook for systematic reviews of interventions. Hoboken: Wiley-Blackwell, 2008.

30 Practice CE, Organisation of Care. Cochrane data abstraction form. EPOC resources for review authors, 2013. Available: https://epoc. cochrane.org/resources/epoc-resources-review-authors [Accessed 10 June 2020].

31 Sterne JAC, Savović J, Page MJ, et al. Rob 2: a revised tool for assessing risk of bias in randomised trials. BMJ 2019;366:14898.

32 Popay J, Roberts H, Sowden A, et al. Narrative synthesis in systematic reviews: a product from the ESRC methods programme. ESRC Methods Programme 2006;2006:93.

33 Akl EA, Briel M, You JJ, et al. Potential impact on estimated treatment effects of information lost to follow-up in randomised controlled trials (LOST-IT): systematic review. BMJ 2012;344:e2809.

34 McLean S, Holden MA, Potia T, et al. Quality and acceptability of measures of exercise adherence in musculoskeletal settings: a systematic review. Rheumatology 2017;56:426-38.

35 World Health Organization. Adherence to long-term therapies: evidence for action. Geneva: World Health Organization, 2003: 198. 